\title{
Clinics in diagnostic imaging (184)
}

Dinesh $R$ Singh $^{1}$, MMed, FRCR, Ashish Chawla ${ }^{1}$, DABR, Wilfred CG $\underline{P e h}^{1}$, FRCP, FRCR

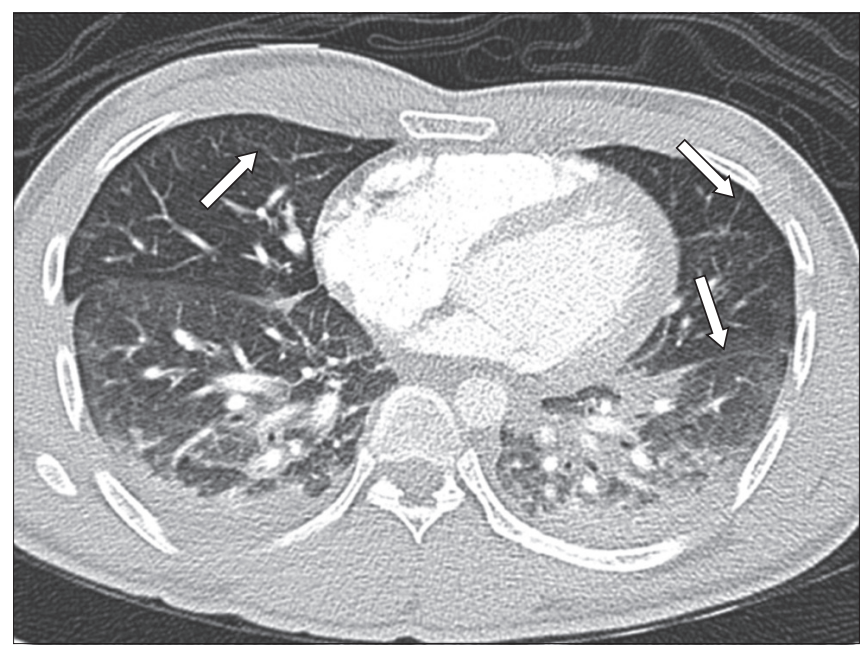

Fig. 1 Contrast-enhanced axial CT image (lung window).
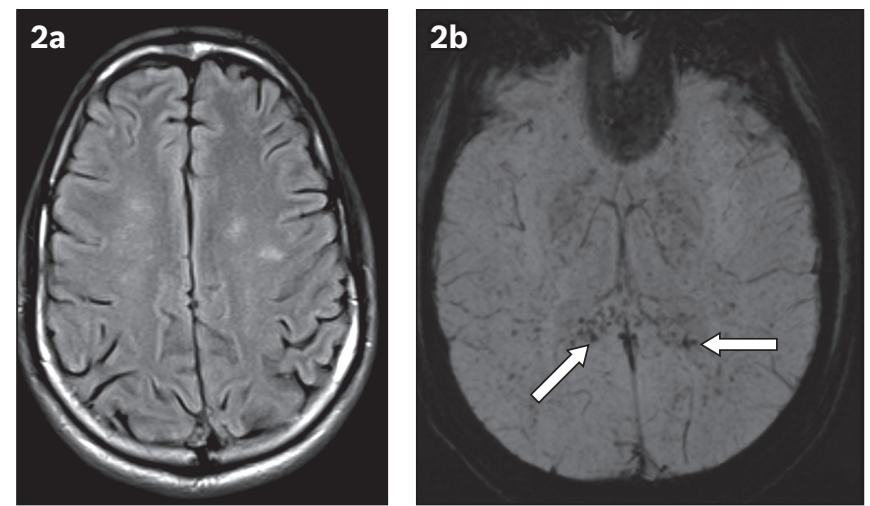

Fig. 2 Axial MR (a) fluid-attenuated inversion recovery (FLAIR) and (b) susceptibility-weighted (SW) images.

\section{CASE PRESENTATION}

A 23-year-old Indian man presented to the emergency department following an injury to his right leg. Radiographs showed a fracture of the midshaft of the right femur, for which open reduction and internal fixation was subsequently performed. The patient developed shortness of breath on Postoperative Day 2, along with new-onset confusion and altered mental state. His lungs were clear on the clinical examination, but there was a new rash on the chest. The patient's condition rapidly deteriorated over the next few hours with progressive worsening of the hypoxia, and he had to be intubated. He also experienced rapid neurological decline with a worsening score on the Glasgow Coma Scale (GCS) from 15 to 11. Blood tests demonstrated mild anaemia with thrombocytopenia. Computed tomography (CT) pulmonary angiography (Fig. 1) was performed to evaluate the cause of shortness of breath, and magnetic resonance (MR) imaging of the brain was performed to assess the rapid neurological decline (Fig. 2). What do the images show? What is the diagnosis? 


\section{IMAGE INTERPRETATION}

The CT axial image of the lung window (Fig. 1) shows changes of mosaic attenuation in both lungs with smooth interlobular septal thickening (arrows) and bilateral pleural effusions. There was no demonstrable filling defect involving the pulmonary arteries or their major divisions (not shown). MR imaging of the brain shows patchy fluid-attenuated inversion recovery (FLAIR) signal abnormalities in the periventricular, deep and subcortical white matter (Fig. 2a) along with innumerable tiny, discrete hypointense foci (arrows, Fig. 2b) on the susceptibilityweighted (SW) images. These susceptibility foci, predominantly involving the white matter regions, are in keeping with petechial haemorrhages (Fig. 2b).

\section{DIAGNOSIS}

Fat embolism syndrome (FES).

\section{CLINICAL COURSE}

A diagnosis of FES was established on the basis of a combination of the clinical, radiological and laboratory findings. Two-dimensional echocardiography was unremarkable. Cerebrospinal fluid (CSF) analysis and CSF virology were negative. There was gradual improvement in the patient's condition over the next week, with improvement in the GCS score (from 11 to 15). The patient was successfully extubated and transferred to the general ward. His platelet count improved as well, with some thrombocytosis following surgery, which was attributed to reactive thrombocytosis. The patient was able to ambulate independently with the help of crutches and was subsequently discharged.

\section{DISCUSSION}

FES is a well-described syndrome that was first reported in 1962.(1) It is classically associated with fracture of the long bones and is most often seen within the first three days, after the injury. ${ }^{(2)}$ FES is a combination of central nervous system (CNS), respiratory and cutaneous manifestations, with incidence rates ranging from $0.9 \%$ to $2.2 \%$. ${ }^{(1)}$ FES can vary in severity and, in some cases, even lead to death, with mortality rates of $13 \%$ to $87 \%$. ${ }^{(3)}$ Subclinical fat embolism, seen in almost all cases of fractured long bones, is asymptomatic, self-limiting and does not warrant any treatment.

FES occurs due to dissemination of fatty tissue from the bone marrow as a result of the long-bone fracture. This disseminated marrow fat tissue can get lodged in the pulmonary circulation, leading to respiratory symptoms, typically acute-onset shortness of breath, and hypoxia. The respiratory manifestations are the first to occur and may be followed by neurological and skin findings. The marrow fat emboli can escape to the systemic circulation in patients with right-to-left cardiac shunts. As most patients with FES have no demonstrable cardiac shunts, it has been proposed that small fat vacuoles $(<5 \mu \mathrm{m})$ can directly pass from the pulmonary to the systemic circulation. ${ }^{(1)}$ Showers of fat emboli can occur in the brain and occlude small capillaries, causing ischaemia. Typical neurological complaints include headache, acute-onset confusion, disorientation, weakness, convulsions and even coma. Typical lesions of cerebral fat embolism (CFE) involve white matter with relative sparing of grey matter, as the grey matter is rich in vascularity. CNS lesions may be due to ischaemia or secondary to haemorrhages. The white matter lesions occur mainly due to venous sludging and oedema. ${ }^{(1)}$

There is usually a time lag between the pulmonary and CNS symptoms whose time duration depends on the filter effect (time taken for the passage of emboli from the pulmonary to the systemic circulation). The onset of neurological symptoms is therefore faster and much worse in patients with right-to-left shunts, such as in cases of patent foramen ovale. Neurological recovery depends on the severity of involvement, with some cases leading to brain atrophy and demyelinating changes. However, most neurological issues that occur are transient and often completely reversible. The cutaneous manifestations usually appear after 24 hours and usually disappear within a week. The classical skin manifestation is a petechial rash, typically appearing on the chest, neck, axilla or even the conjunctiva. The rash resolves spontaneously within a few days.

FES is a diagnostic challenge for the clinician. One set of commonly used criteria for diagnosis is by Gurd and Wilson, which states that the clinical diagnosis of FES can be made if at least two major or one major and at least four minor points are met. ${ }^{(4)}$ Major criteria include petechial rash, respiratory insufficiency and cerebral involvement. Minor criteria include tachycardia, fever, retinal changes, jaundice, renal signs, thrombocytopenia, anaemia, fat macroglobulinaemia and high erythrocyte sedimentation rate.

Imaging plays an extremely important role in evaluating patients with suspected FES. Chest radiographs can be nonspecific, ranging from the lack of obvious findings to patchy ground-glass opacities. Typical lesions on CT of the thorax include geographic ground-glass opacities, interlobular septal thickening or even nodular opacities. ${ }^{(5,6)}$ Filling defects within the pulmonary arteries are rarely seen. ${ }^{(5)}$ CT of the brain can often be negative or may show subtle hypodense foci, which are non-specific. MR imaging is the diagnostic modality of choice in evaluations of patients with CFE. ${ }^{(7)}$ Findings include patchy or confluent oedema changes, sometimes with restricted diffusion and enhancement on postcontrast evaluation. The lesions usually involve the watershed zone, deep grey matter and white matter; less commonly, there is involvement of the corpus callosum, cerebral peduncles and posterior internal capsule. There may also be petechial haemorrhages in the white matter distribution, as identified on gradient-echo and SW images. ${ }^{(8,9)}$ The patchy lesions in the watershed zone and the deep grey matter have also been described as a 'starfield pattern' in 2001, by Parizel et al. ${ }^{(10)}$

In summary, fat embolism syndrome is a challenging clinical diagnosis that is classically seen in patients following long-bone fractures. Although most cases are subclinical, some can lead to death, especially in the presence of neurological and respiratory complications. MR imaging findings can have overlapping features with other conditions such as hypoxic-ischaemic injury (Fig. 3a-c), diffuse axonal injury (Fig. 4a-c), metabolic encephalopathy or even haemorrhagic metastasis, and diagnosis is based on a combination of clinical, imaging and laboratory findings. 

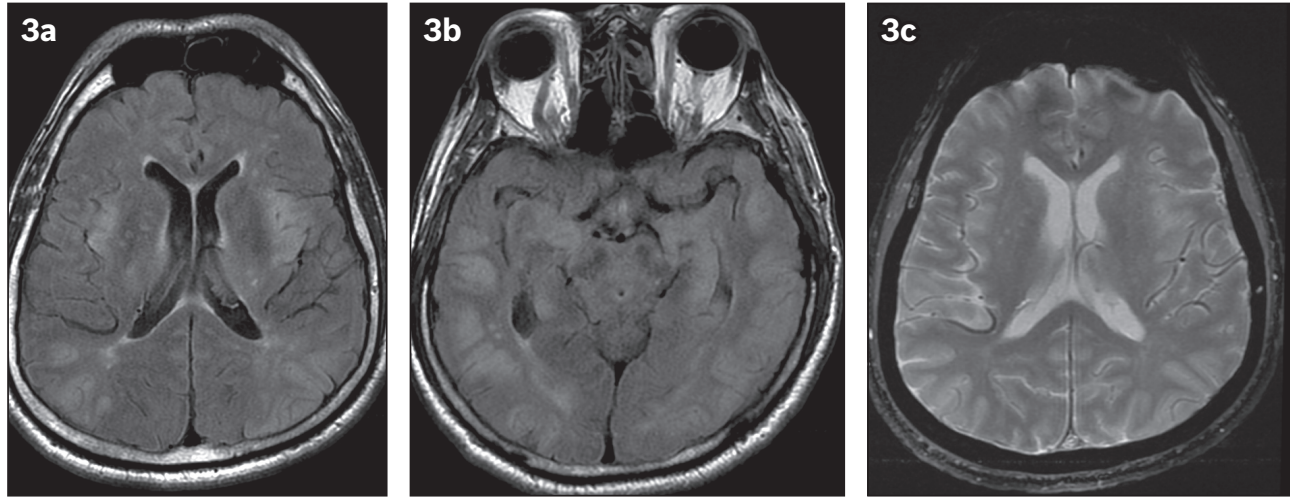

Fig. 3 A 42-year-old man found collapsed by the roadside received prolonged resuscitation and was rushed to the emergency department. (a \& b) FLAIR MR images show extensive oedema changes, predominantly in the grey matter structures. (c) SW images show no haemorrhage. The imaging findings are consistent with hypoxic-ischaemic injury.
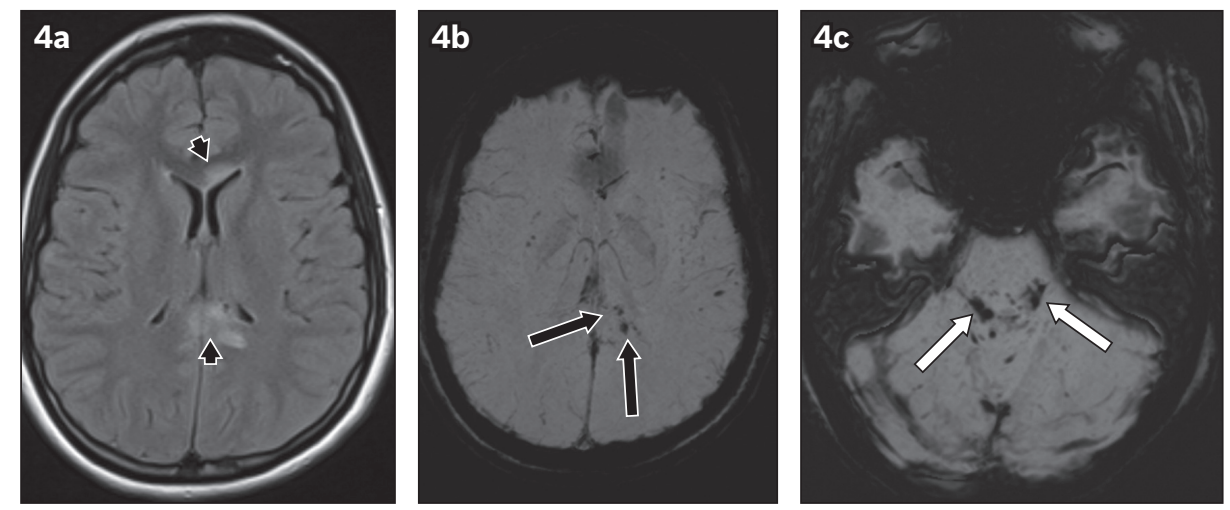

Fig. 4 A 27-year-old man presented at the emergency department with a low Glasgow Coma Scale score following a road traffic accident. Axial (a) FLAIR and (b \& c) SW images show oedematous lesions (black arrowheads, Fig. 4a) and haemorrhagic lesions involving the corpus callosum (black arrows, Fig. 4b), petechial haemorrhages at the grey-white matter junction region and mild basal subarachnoid haemorrhage (white arrows, Fig 4c). The findings are consistent with changes from diffuse axonal injury.

ABSTRACT A 23-year-old Indian man presented with shortness of breath and new-onset confusion along with a rash on his chest on Postoperative Day 2, following internal fixation of his femur fracture. Although computed tomography pulmonary angiography was negative for filling defects in the pulmonary vasculature, it showed mosaic attenuation changes with some interlobular septal thickening. Magnetic resonance imaging of the brain showed patchy signal abnormalities, predominantly in the grey-white matter junction region with extensive susceptibility artefacts, consistent with petechial haemorrhages. The laboratory work-up showed thrombocytopenia and anaemia. A diagnosis of fat embolism syndrome was established, based on the clinical presentation combined with laboratory and imaging findings. The clinical and imaging features of fat embolism syndrome are discussed.

Keywords: altered mental state, fat embolism syndrome, hypoxia, petechial haemorrhages

\section{REFERENCES}

1. Kuo KH, Pan YJ, Lai YJ, et al. Dynamic MR imaging patterns of cerebral fat embolism: a systematic review with illustrative cases. AJNR Am J Neuroradiol 2014; 35:1052-7.

2. Aman J, van Koppenhagen L, Snoek AM, van der Hoeven JG, van der Lely AJ. Cerebral fat embolism after bone fractures. Lancet 2015; 386:e16.

3. Simon AD, Ulmer JL, Strottmann JM. Contrast-enhanced MR imaging of cerebral fat embolism: case report and review of the literature. AJNR Am J Neuroradiol 2003; 24:97-101.

4. Gurd AR, Wilson RI. The fat embolism syndrome. J Bone Joint Surg Br 1974; 56:408-16.

5. Piolanti M, Dalpiaz G, Scaglione $M$, et al. Fat embolism syndrome: lung computed tomography findings in 18 patients. J Comput Assist Tomogr 2016; 40:335-42.

6. Newbigin K, Souza CA, Torres C, et al,. Fat embolism syndrome: state-of-the-art review focused on pulmonary imaging findings. Respir Med 2016; 113:93-100.

7. Chen JJ, Ha JC, Mirvis SE. MR imaging of the brain in fat embolism syndrome. Emerg Radiol 2008; 15:187-92.

8. Mossa-Basha M, Izbudak I, Gurda GT, Aygun N. Cerebral fat embolism syndrome in sickle cell anaemia/ $\beta$-thalassemia: importance of susceptibility-weighted MRI. Clin Radiol 2012; 67:1023-6.

9. Zaitsu Y, Terae S, Kudo K, et al. Susceptibility-weighted imaging of cerebral fat embolism. J Comput Assist Tomogr 2010; 34:107-12.

10. Parizel PM, Demey HE, Veeckmans G, et al. Early diagnosis of cerebral fat embolism syndrome by diffusion-weighted MRI (starfield pattern). Stroke 2001; 32:2942-4. 


\section{SINGAPORE MEDICAL COUNCIL CATEGORY 3B CME PROGRAMME} (Code SMJ 201803B)

Question 1. Regarding diagnosis of fat embolism syndrome (FES):

(a) It is purely clinical.

(b) It is purely radiological.

(c) It can be challenging for clinicians.

(d) It is based on a combination of clinical, radiological and laboratory findings.

Question 2. Regarding FES:

(a) Cutaneous manifestations are usually the first to occur.

(b) Respiratory manifestations are usually the first to occur.

(c) Central nervous system manifestations always precede respiratory manifestations.

(d) Acute-onset shortness of breath is a typical respiratory manifestation.

Question 3. Regarding cutaneous manifestations in FES:

(a) They typically occur after a delay of 2-3 weeks.

(b) Petechial rash is the typical manifestation.

(c) Aggressive treatment is often required.

(d) They usually resolve spontaneously.

Question 4. Regarding MR imaging findings in cerebral fat embolism:

(a) Lesions may be patchy or confluent.

(b) Lesions rarely involve white matter.

(c) Petechial haemorrhages can be seen.

(d) Lesions can show restricted diffusion.

Question 5. Regarding computed tomography (CT) imaging in FES:

(a) CT brain findings are always diagnostic.

(b) CT pulmonary angiograms often show filling defects in the pulmonary arteries.

(c) Typical CT thorax findings include areas of cavitation and consolidation.

(d) Typical CT thorax findings include ground-glass densities, interlobular septal thickening and sometimes nodules.

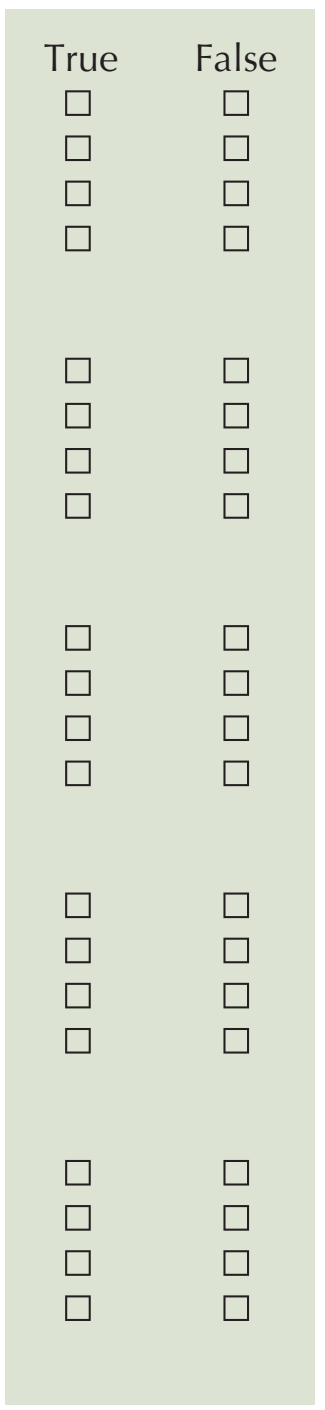

Doctor's particulars:

Name in full

MCR number

Email address

SUBMISSION INSTRUCTIONS:

Visit the SMJ website: http://www.smj.org.sg/current-issue and select the appropriate quiz. You will be redirected to the SMA login page.

For SMA member: (1) Log in with your username and password (if you do not know your password, please click on 'Forgot your password?'). (2) Select your answers for each quiz and click 'Submit'.

For non-SMA member: (1) Create an SMJ CME account, or log in with your SMJ CME username and password (for returning users). (2) Make payment of SGD 21.40 (inclusive of $7 \%$ GST) via PayPal to access this month's quizzes. (3) Select your answers for each quiz and click 'Submit'.

RESULTS:

(1) Answers will be published online in the SMJ May 2018 issue. (2) The MCR numbers of successful candidates will be posted online at the SMJ website by 7 May 2018. (3) Passing mark is $60 \%$. No mark will be deducted for incorrect answers. (4) The SMJ editorial office will submit the list of successful candidates to the Singapore Medical Council. (5) One CME point is awarded for successful candidates.

Deadline for submission: (March 2018 SMJ 3B CME programme): 12 noon, 30 April 2018. 